\title{
Isovaleric acidemia: A rare cause of isolated cerebellar atrophy
}

\author{
Nicolas-Jilwan $\mathbf{M}^{*}$ \\ Department of Radiology, King Faisal Specialist Hospital and Research Centre, Riyadh, Saudi Arabia
}

\begin{abstract}
Isovaleric acidemia is a very rare autosomal recessive inborn error of leucine metabolism caused by deficiency of the mitochondrial enzyme isovaleryl-coenzyme A dehydrogenase. The disease has two well-known clinical phenotypes: an acute neonatal presentation with severe metabolic crisis and a chronic intermittent form. There are only two reported cases of the neuroimaging findings of this disorder, both imaged during an acute metabolic decompensation, with documented abnormalities of the basal ganglia. We report a previously undescribed neuroimaging finding of isolated cerebellar atrophy with normal basal ganglia in a 6-year-old female with chronic intermittent isovaleric acidemia, imaged for evaluation of developmental delay, outside an episode of a metabolic crisis.
\end{abstract}

\section{Introduction}

Isovaleric acidemia is a very rare autosomal recessive inborn error of leucine metabolism caused by a deficiency of the mitochondrial enzyme isovaleryl-coenzyme A dehydrogenase, leading to accumulation of isovaleric acid, a catabolite of leucine [1,2]. The encoding gene is located on chromosome 15 [3]. The disease has two well-known clinical phenotypes: an acute neonatal presentation in the first two weeks of life with vomiting, lethargy and metabolic acidosis that can progress to coma and death and a chronic intermittent phenotype presenting with failure to thrive and/or developmental delay with or without intermittent acute episodes of acidotic decompensation due to intercurrent catabolic stress [2,4]. In both forms, a characteristic feature is the "sweaty feet odor" of the child, particularly noticeable regarding the cerumen and sweat, caused by the accumulated isovaleric acid during episodes of acute decompensation.

There are only two reports $[5,6]$ of abnormal neuroimaging features of isovaleric acidemia in the literature, both describing abnormalities of the basal ganglia, as expected with most organic acidurias. We report a previously undescribed neuroimaging finding of isolated cerebellar atrophy with normal basal ganglia in a 6-year-old female with chronic intermittent isovaleric acidemia.

\section{Case report}

The patient is currently an 8-year-old girl, born full term to consanguineous Saudi parents. She presented with severe metabolic crisis at one week of age, which evolved into coma, adequately treated and reversed. She had subsequent multiple admissions during infancy with recurrent metabolic crisis, poor feeding, vomiting and fever. Tandem mass spectrometry was compatible with isovaleric acidemia, and this was confirmed with mutation analysis. The patient was maintained on a protein restricted diet with L-carnitine and glycine supplementation. The episodes of metabolic decompensation became rare during childhood. However, the patient suffered severe receptive and expressive language delay for which she underwent a brain MRI at 6 years of age (Figure 1). This revealed global cerebellar atrophy of the vermis and hemispheres with normal T2 signal of the cerebellar cortex.
The basal ganglia and thalami were normal in size and signal. White matter myelination was normal. There was otherwise no volume loss of the cerebral hemispheres or brainstem. Single voxel MR spectroscopy using low, intermediate and high TE at the level of the basal ganglia was normal.

\section{Discussion}

There are only two reports available in the literature of abnormal neuroimaging findings of isovaleric acidemia $[5,6]$. Both reported cases were imaged during metabolic decompensation. Wani et al. [5] report signal abnormality in the lentiform nuclei in a 4-month-old girl in metabolic crisis. The case reported by Sogut et al. [6] is a 19-month-old girl in metabolic crisis, with T2 hyperintense signal abnormality in the globi pallidi and mesencephalon on brain MRI as well as global cerebral atrophy with thinning of the corpus callosum. The findings are not unexpected given the known selective vulnerability of the basal ganglia to the oxidative stress of decompensated organic acidurias. Our patient was imaged at an older age of 6 years, outside an episode of metabolic crisis, for evaluation of developmental delay, mainly in the form of severe receptive and expressive speech delay. Her MRI did not show established injury of the basal ganglia, which correlated with the clinical absence of movement disorders. There was however global cerebellar atrophy of the cerebellar hemispheres and vermis. As isovalerylcoenzyme A dehydrogenase is a mitochondrial enzyme and given that mitochondrial disorders are the most common cause of cerebellar atrophy [7], the finding of cerebellar atrophy in isovaleric acidemia is not surprising. The susceptibility of the cerebellum to selective injury with organic acidurias, such as in particular 3-methylglutaconic aciduria [7], is another explanation of the cerebellar atrophy seen in our

*Correspondence to: Nicolas-Jilwan M, Department of Radiology, King Faisal Specialist Hospital and Research Centre, Riyadh, Saudi Arabia, Tel: 9613940781; E-mail: manaljilwan@hotmail.com

Key words: Isovaleric acidemia, basal ganglia, cerebellar atrophy, organic aciduria. Received: June 27, 2018 Accepted: July 06, 2018; Published: July 09, 2018 

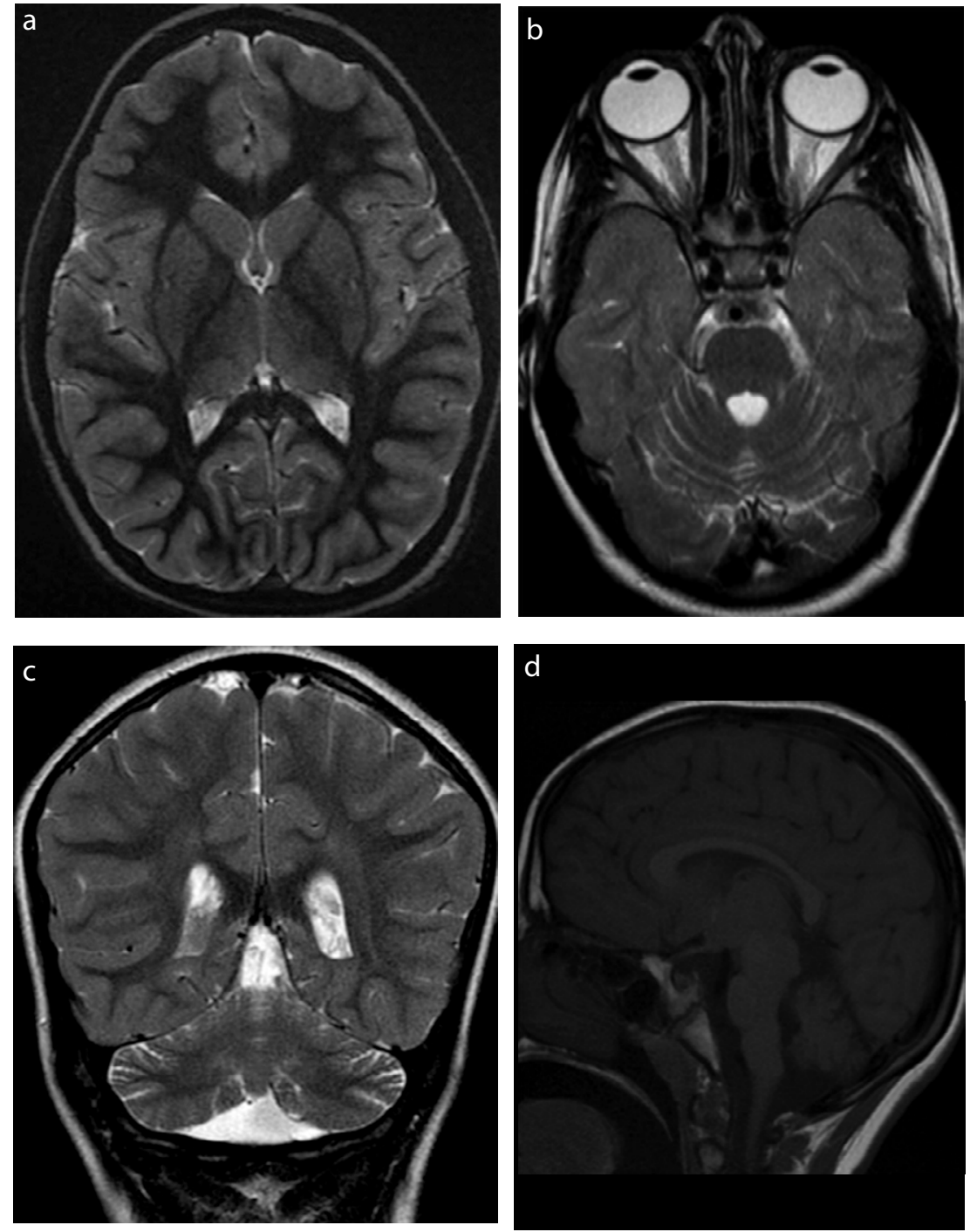

Figure 1. a) Axial Inversion Recovery image (TR/TE 5250/50.9 ms) demonstrates normal signal and size of the basal ganglia. b) Axial Inversion Recovery image (TR/TE 5250/50.9 ms) shows mild widening of the cerebellar sulci and fourth ventricle indicative of mild cerebellar atrophy, further confirmed on c) coronal T2 image (TR/TE $3583 / 99 \mathrm{~ms}$ ) and d) Sagittal T1 image (TR/TE 350/9 ms)

patient. The finding of cerebellar atrophy in our patient is a potential imaging explanation of the mild dyscoordination which can be seen in patients with isovaleric acidemia [4].

\section{Conclusion}

Our case demonstrates that isovaleric acidemia is one rare etiology of isolated cerebellar atrophy in children. This hopefully represents a small contribution toward better characterization of childhood cerebellar atrophy which remains without a specific diagnosis in over $50 \%$ of cases [7].

\section{References}

1. Tanaka K, Budd MA, Efron ML, Isselbacher KJ (1966) Isovaleric acidemia: a new genetic defect of leucine metabolism. Proc Natl Acad Sci U S A 56: 236-242. [Crossref]

2. Vockley J, Ensenauer R (2006) Isovaleric acidemia: new aspects of genetic and phenotypic heterogeneity. Am J Med Genet C Semin Med Genet 142C: 95-103. [Crossref]

3. Parimoo B, Tanaka K (1993) Structural organization of the human isovaleryl-CoA dehydrogenase gene. Genomics 15: 582-590. [Crossref]
4. Grünert SC, Wendel U, Lindner M, Leichsenring M, Schwab KO, et al. (2012) Clinical and neurocognitive outcome in symptomatic isovaleric acidemia. Orphanet J Rare Dis 7:9.

5. Wani NA, Qureshi UA, Jehangir M, Ahmad K, Hussain Z (2016) Atypical MR lenticular signal change in infantile isovaleric acidemia. Indian J Radiol Imaging 26 : 131-134. [Crossref]

6. Sogut A, Acun C, Aydin K, Tomac N, Demirel F, et al. (2004) Isovaleric acidaemia: cranial CT and MRI findings. Pediatr Radiol 34: 160-162. [Crossref]

7. Al-Maawali A, Blaser S, Yoon G (2012) Diagnostic approach to childhood-onse cerebellar atrophy: a 10-year retrospective study of 300 patients. J Child Neurol 27: 1121-1132. [Crossref]

Copyright: (C2018 Nicolas-Jilwan M. This is an open-access article distributed under the terms of the Creative Commons Attribution License, which permits unrestricted use, distribution, and reproduction in any medium, provided the original author and source are credited. 